\title{
Semantic Feature Analysis: Further Examination of Outcomes
}

Semantic Feature Analysis (SFA) has received considerable study over the past two decades as a word-retrieval treatment for aphasia (Boyle \& Coelho, 1995; Lowell, Beeson, \& Holland, 1995; Boyle, 2010; Wambaugh, Mauszycki, Cameron, Wright, \& Nessler, 2013). SFA has been shown to have consistently positive acquisition effects (i.e., improvement of trained items), with generally positive but less predictable generalization effects (i.e., improvement in untrained items).

SFA was originally designed as a cognitive treatment for children and adolescents sustaining traumatic brain injury (TBI) (Haarbauer-Krupa, Moser, Smith, Sullivan \& Szekeres, 1985). The therapy was designed as an "organizing process for thinking and verbal expression" (p.303).

Massaro and Tompkins (1994) operationalized SFA in a study with two participants with TBI. In keeping with the intentions of the original developers, Massaro and Tompkins measured SFA's treatment effects in terms of increased production of semantically relevant content.

In the treatment of aphasia, the focus of SFA relative to outcomes has been naming accuracy. That is, SFA has been used as a means of systematically stimulating semantic networks to facilitate naming. Additionally, SFA has been considered to potentially serve as a mediating strategy for self-cuing accurate naming and/or a compensatory strategy for circumventing word-retrieval difficulties.

The current study was designed to elucidate the effects of SFA in aphasia treatment beyond naming accuracy. Given SFA was designed to improve verbal expression in general and may serve as a compensatory strategy, increased production of relevant content was of interest (after Tompkins \& Massaro, 1994). In light of inconsistent generalization effects associated with SFA, the study was designed to explore its generalization effects relative to aspects of untreated items. Specifically, untreated items were controlled in terms of semantic relatedness, exposure in probing, and knowledge of phonological form.

\section{Method}

\section{Participants}

Four persons with chronic aphasia resulting from a single, left-hemisphere stroke (Table 1) served as participants. As seen in Table 2, participants were each diagnosed with a different aphasia type according to the Western Aphasia Battery (WAB; Kertesz, 1982).

\section{Experimental Stimuli}

Three sets of picture stimuli were created for each participant Sets 1 and 2 contained 32 items each, and Set 3 contained eight items (Appendix B). Each set represented two different semantic categories; one living and one non-living. Eight treatment items from Sets 1 and 2 were submitted to SFA during the designated treatment phase; the remaining items were used to measure generalization.

\section{Experimental Design}

A multiple baseline design across behaviors and participants was utilized to examine the effects of treatment on naming and production of semantically appropriate information. Naming 
of items used as treatment, generalization, and pre/post measures were probed repeatedly in the baseline phase. Three "information probes" were conducted prior to treatment to measure production of semantically relevant content. Treatment was then applied sequentially to two sets of experimental items.

\title{
Baseline Phase
}

Sixteen treatment items, 40 generalization items, and 16 pre/post items were probed during each baseline session. The number of baseline probes was extended across participants, with five as the minimum. Information probes contained two items from each list and category, totaling 36 items per probe. Three information probes were conducted in baseline.

\section{Treatment Phase}

Treatment probes were conducted at the beginning of each session prior to treatment. The eight treatment items were randomized and probed after every two treatment sessions. The sixteen generalization exposure control items were probed at the beginning of every other session when not probing treatment items. During the treatment phases, the second set of items (treatment and generalization items) was probed approximately half way through the first phase, and repeatedly prior to initiating the second phase.

Information probes were conducted at the end of each treatment phase.

\section{Maintenance and Follow-up Phases}

The previously treated set (treatment and generalization items) was probed half way, and at the end of the second treatment phase. Follow-up probes for all lists and items were conducted at two and six weeks after treatment ended.

\section{Probe Procedures and Dependent Variable}

Probes were conducted repeatedly throughout all phases of the design in keeping with single-subject design conventions.

Confrontation Naming Probes. Performance on naming probes served as the basis for determining phase changes of the design. During baseline and treatment probes, each item was presented one at a time, for verbal naming in random order.

Naming responses were scored as correct or incorrect on the basis of the scoring system shown in Appendix C. Responses were judged on the first complete production within the allotted 20 seconds.

Semantic Information Probes. Semantic information probes were administered to examine amount of relevant semantic information provided about target items. The participant was allowed two minutes to respond. Words adding relevant and novel content to an item's description were counted as semantic information units (SIUs). Task instructions were, "I'm going to show you pictures one at a time. Instead of naming the picture, think about the picture and tell me as much as you can about it.” Word counts were tabulated using Nicholas and Brookshire (1993) procedures. The SIU measure differed from Nicholas and Brookshire's Correct Information Units (CIU) in that SIUs excluded articles, auxiliary verbs, and conjunctions. Example transcriptions with SIUs underlined are shown in Appendix D.

\section{Treatment}

\author{
Semantic Feature Analysis Task
}


Treatment consisted of Sematic Feature Analysis as described by Boyle (2004).

However, slight modifications to the SFA chart were made to accommodate living and nonliving categories.

\section{Exposure Control/Phonological Form Task}

Because repeated attempts at naming may result in improved naming in the absence of treatment (Howard, 2000) a set of items was presented for naming during the treatment session so that naming exposures were relatively equivalent; no feedback concerning naming accuracy was provided. In addition, improvements in semantic network access/organization may be masked by remaining phonological level processing deficits in some persons with aphasia. Consequently, another set of items was presented for naming during treatment and the correct name was provided in the event of inaccurate naming and feedback was provided for correct naming.

\section{Results}

The data representing naming accuracy during probes are shown in Figures 1-4. Effect sizes (d-index,: Bloom, Fischer, \& Orme, 2003; Cohen, 1998) were calculated to measure the magnitude of change associated with treatment (Table 3). Based on Beeson and Robey (2006) benchmarks for interpreting effect sizes, trained items ranged from small effects, 4.04 to large effects, 14.52. Generalization items for which participants were exposed to the phonological forms, showed small to medium effects. Generalization items that did not have phonological exposure had small to medium effects, 7.51. Effect sizes for pre/post items were small showing limited generalization. Each participant increased the amount semantic information provided during information probes, as shown in Figure 5. Note: Participant 4 will complete the second phase of treatment within two weeks of this submission.

\section{Discussion}

In this study, SFA facilitated increases in naming accuracy of treated items. Generalization to untreated items when controlled for semantic relatedness, exposure during probes, and knowledge of phonological form, was mixed and findings will be discussed further in relation to participant characteristics. Further use of SFA as a compensatory strategy to provide semantic information could be beneficial, in addition it's use as a naming treatment. 


\section{References}

Beeson, P. M., \& Robey, R. R. (2006). Evaluating single subject treatment research: Lessons learned from the aphasia literature. Neuropsychology Review, 16(4), 161-169.

Bloom, M., Fisher, J., \& Orme, J. G. (2003) Evaluating practice-Guidelines for the accountable professional ( $4^{\text {th }}$ ed.) Boston: Allyn \& Bacon, Pearson Education, Inc.

Boyle, M., \& Coelho, C. (1995) Application of semantic feature analysis as a treatment for aphasic dysnomia. American Journal of Speech-Language Pathology, 4, 94-98.

Boyle, M. (2004) Semantic feature analysis treatment for anomia in two fluent aphasia syndromes. American Journal of Speech-Language Pathology, 13, 236-249.

Boyle, M. (2010). Semantic feature analysis treatment for aphasic word retrieval impairments: what's in a name? Topics in Stroke Rehabilitation, 17(6), 411-422.

Brown, L., Serbenou, R.J., \& Johnsen, S.K. (2010). Test of Nonverbal Intelligence - 4. Austin, TX: Pro-ed.

Cohen, J. (1998). Statistical power analysis for the behavioral sciences ( $2^{\text {nd }}$ ed.). Hillsdale, NJ: Erlebaum.

Drunks, J. \& Masterson, J. (2000). An object and action naming battery. Hove, UK: Psychology Press.

German, D. J. (1990). Test of adolescent/adult word finding. Allen, TX: DLM.

Haarber-Krupa, J., Moser, L., Smith, G., Sullivan, D. M., \& Szekeres, S. F. (1985). Cognitiverehabilitative therapy: Middle stages of recovery. In M. Ylvisaker (Ed). Head injury rehabilitation: Children and adolescents (pp.287-310). San Diego, CA: College-Hill Press.

Howard, D. and Patterson, K. (1992). The Pyramids and Palm Trees Test. Thames Valley Test Company.

Howard, D. Cognitive neuropsychology and aphasia therapy: the case of word retrieval. In: Papathanasiou I, ed. Acquired Neurogenic Communication Disorders: A Clinical Perspective. London, England: Whurr; 2000: 76-79.

Kay, J., Lesser, R., \& Coltheart, M. (1992). The Psycholinguistic Assessment of Language Processing in Aphasia (PALPA), Hove, United Kingdom: Erlbaum.

Kertesz, A. (1982). The Western Aphasia Battery. New York: Grune \& Stratton.

Lowell, S., Beeson, P., \& Holland, A. (1995) Semantic cueing procedure on naming performance of adults with aphasia. American Journal of Speech-Language Pathology, 4, 109-114.

Massaro, M., \& Tompkins, C. A. (1994). Feature analysis for treatment of communication disorders in traumatically brain-injured patients: an efficacy study [Electronic version]. Clinical Aphasiology, 22, 245-256.

Nicholas, L. E., \& Brookshire, R. H. (1993, April). A system for quantifying the informativeness and efficiency of the connected speech of adults with aphasia. Journal of speech and hearing research, 36, 338-350.

Porch, B. (2001). Porch index of communicative ability (Vol.2). Administration, scoring and interpretation ( $4^{\text {th }}$ ed.). Albuquerque, NM: PICA Programs.

Wambaugh, J. L., Mauszycki, S., Cameron, R., Wright, S., \& Nessler, C. (2013, May). Semantic feature analysis: incorporating typicality treatment and mediating strategy training to promote generalization. American Journal of Speech-Language Pathology, 22(2), 334369. 
Table 1.

Participant Characteristics

\begin{tabular}{lcccc}
\hline Characteristic & Participant 1 & Participant 2 & Participant 3 & Participant 4 \\
\hline Age & 62 & 54 & 30 & 53 \\
Gender & female & male & male & female \\
MPO & 11 & 30 & 23 & 384 \\
CVA Location & LCVA & LMCA & LMCA & LMCA \\
Type & carotid dissection & ischemic & ischemic & ischemic \\
Years of & & & & \\
Education & 12 & 16 & 12 & 16 \\
Race/Ethnicity & White & White & White & White \\
& non H/L & non H/L & non H/L & non H/L \\
$\begin{array}{l}\text { Handedness } \\
\text { (premorbid) }\end{array}$ & right & right & right & right \\
Martial Status & married & single & married & married \\
\hline
\end{tabular}

$\mathrm{L}=$ left; $\mathrm{R}$ = right; MCA = middle cerebral artery; PCA= posterior cerebral artery; $\mathrm{H} / \mathrm{L}=$ Hispanic/latino 
Table 2.

Pretreatment assessment results

\begin{tabular}{lcccc}
\hline Assessment & Participant 1 & Participant 2 & Participant 3 & Participant 4 \\
\hline TONI-4 & & & & \\
Index Score & 118 & 109 & 104 & 113 \\
SEM & 3 & 3 & 3 & 3 \\
Percentile & 88 & 73 & 61 & 81
\end{tabular}

WAB

Aphasia Quotient

64.5

59.88

66.1

78.4

Aphasia Type

Conduction

Wernicke's

Broca's

Anomic

AQ Totals:

Spontaneous speech

12

12

13

17

Comprehension

9.05

7.34

6.75

8.3

Repetition

5.2

Naming

6

5.2

6.2

5.5

5.4

7.1

8.4

PICA

Overall Percentile

62nd

$48^{\text {th }}$

Verbal Percentile

Auditory Percentile

$89^{\text {th }}$

$53^{\text {rd }}$

$44^{\text {th }}$

$36^{\text {th }}$

$60^{\text {th }}$

$82^{\text {nd }}$

$66^{\text {th }}$

$71^{\text {st }}$

$53^{\text {rd }}$

$63^{\text {rd }}$

Nicholas and Brookshire (1993)-Discourse Task

Total time

Total \# words

Total \# CIUs

PPT Total (3 picture)

\section{TAAWF}

Total Raw Score

Comprehension

\section{Word Generation for \\ Categories}

Appliances

Body Parts
41

$99 \%$
23:30:00

740

335

$51 / 52$
27

$97 \%$
15

$86 \%$
17:10:00

2,535

1,043

$50 / 52$

23

93\%
3

7
1

10
2

5
11

12 
Fish

Insects

Transportation

Weapons

Category Card Sort

\section{OANB}

Objects

Actions

\section{3}

3

3

1

$70 / 70$

$70 / 70$

$70 / 70$

$70 / 70$

$60 \%$

$78 \%$
$46 \%$

$59 \%$

$65 \%$

$54 \%$

$86 \%$

$56 \%$

\section{PALPA}

Spoken Word-Picture Matching

$39 / 40$

$40 / 40$

$37 / 40$

40/40

Written Word-Picture

$40 / 40$

$40 / 40$

$37 / 40$

$40 / 40$

Matching

Auditory Synonym Judgments

$54 / 60$

$50 / 60$

$54 / 60$

43/60

Word Association

24/30

22/30

10/30

21/30

TONI-4= Test of Nonverbal Intelligence-4 (Brown, Serbenou \& Johnson, 2010); WAB-R= Western Aphasia Battery-R (Kertesz,1982); PICA= Porch Index of Communicative Ability (Porch, 2001); PPT= Pyramids and Palm Trees Test (Howard \& Patterson, 1992); TAAWF= Test of Adolescent/Adult Word Finding (German, 1990); OANB= Object and Action Naming Battery (Drunks \& Masterson, 2000); PALPA= Psycholinguistic Assessment of Language Processing in Aphasia (Kay, Lesser \& Coltheart, 1992) 
Table 3.

Effect Sizes: d-Index Values for Treatment and Follow-Up Phases Interpreted Relative to Robey and Beeson's (2006) Benchmarks

\begin{tabular}{|c|c|c|}
\hline $\begin{array}{l}\text { Participant/ } \\
\text { Experimental Set }\end{array}$ & Baseline - Treatment & Baseline - Follow-up \\
\hline \multicolumn{3}{|l|}{ Participant 1} \\
\hline Treatment Set 1 & 4.04 (small) & 2.68 (small) \\
\hline Generalization-Name & 3.26 & 2.11 \\
\hline Generalization-No Name & 7.51 (medium) & 4.24 \\
\hline Pre/Post & na & .72 \\
\hline Treatment Set 2 & 3.15 & 2.27 \\
\hline Generalization-Name & 1.37 & 3.67 \\
\hline Generalization-No Name & 6.99 (small) & 6.97 (small) \\
\hline Pre/Post & na & 1.99 \\
\hline Generalization Set 3 & na & 2.66 \\
\hline \multicolumn{3}{|l|}{ Participant 2} \\
\hline Treatment Set 1 & 1.43 & 2.39 \\
\hline Generalization-Name & 1.62 & 2.48 \\
\hline Generalization-No Name & 2.88 & 2.00 \\
\hline Pre/Post & na & .22 \\
\hline Treatment Set 2 & 4.32 & 3.47 \\
\hline Generalization-Name & 3.45 & 3.61 \\
\hline Generalization-No Name & 1.31 & 2.83 \\
\hline Pre/Post & na & .50 \\
\hline Generalization Set 3 & na & 1.41 \\
\hline \multicolumn{3}{|l|}{ Participant 3} \\
\hline Treatment Set 1 & 11.24 (large) & 14.52 (large) \\
\hline Generalization-Name & 4.78 & 6.02 \\
\hline Generalization-No Name & 3.69 & 3.44 \\
\hline Pre/Post & na & 1.97 \\
\hline Treatment Set 2 & 6.45 & 4.55 \\
\hline Generalization-Name & 5.0 & 7.64 (medium) \\
\hline Generalization-No Name & 2.83 & 3.62 \\
\hline Pre/Post & na & .08 \\
\hline Generalization Set 3 & na & 1.59 \\
\hline \multicolumn{3}{|l|}{ Participant 4} \\
\hline Treatment Set 1 & 3.18 (small) & na \\
\hline Generalization-Name & 4.49 & na \\
\hline Generalization-No Name & 5.82 & na \\
\hline Pre/Post & na & na \\
\hline Treatment Set 2 & na & na \\
\hline Generalization-Name & na & na \\
\hline
\end{tabular}


Generalization-No Name

Pre/Post

Generalization Set 3 na

na

na na

na

na 


\section{Figure Captions 1-4}

Figure 1: Accuracy of naming of experimental probes for Participant 1 Figure 2: Accuracy of naming of experimental probes for Participant 2 Figure 3: Accuracy of naming of experimental probes for Participant 3 Figure 4: Accuracy of naming of experimental probes for Participant 4 


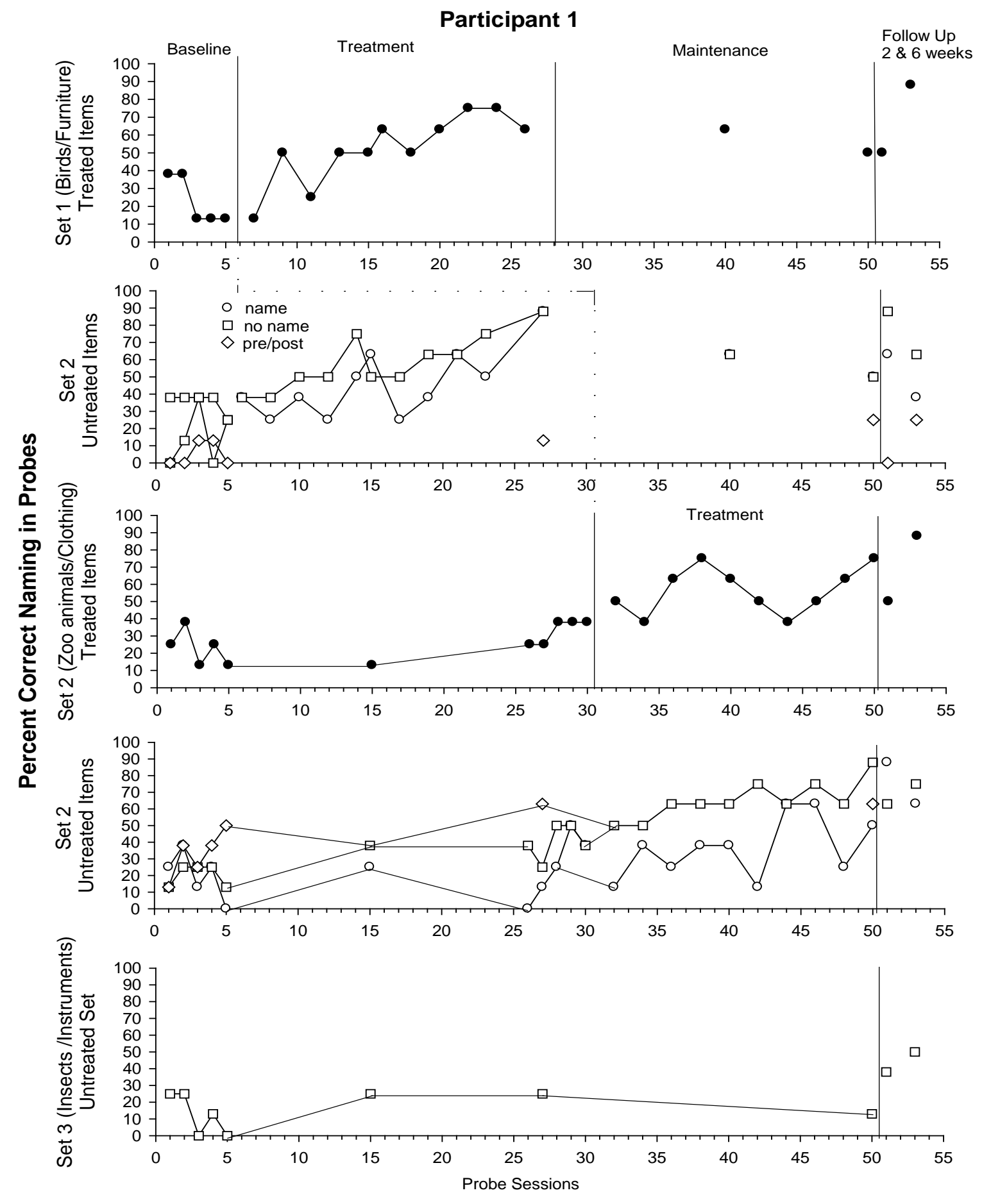


Participant 2
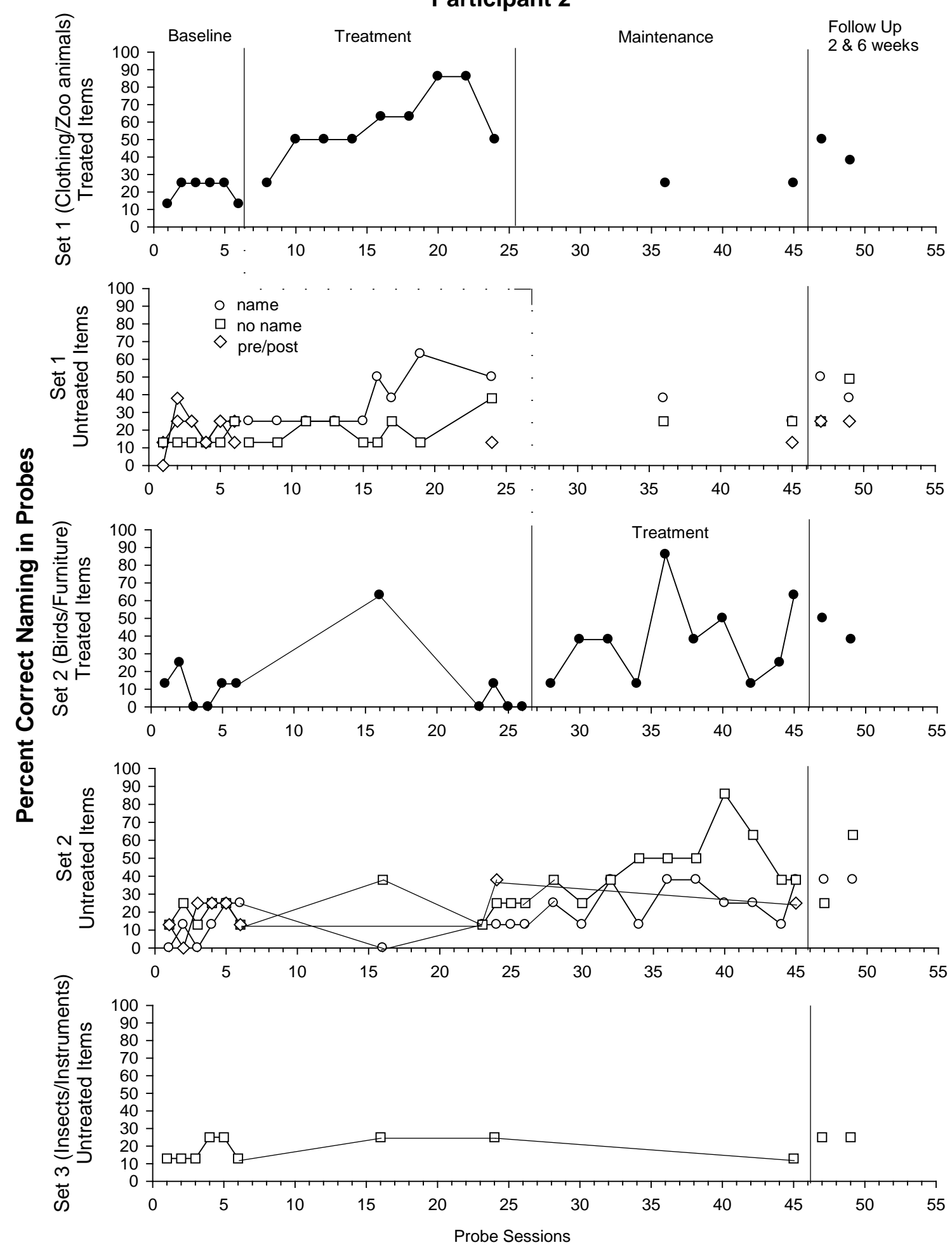

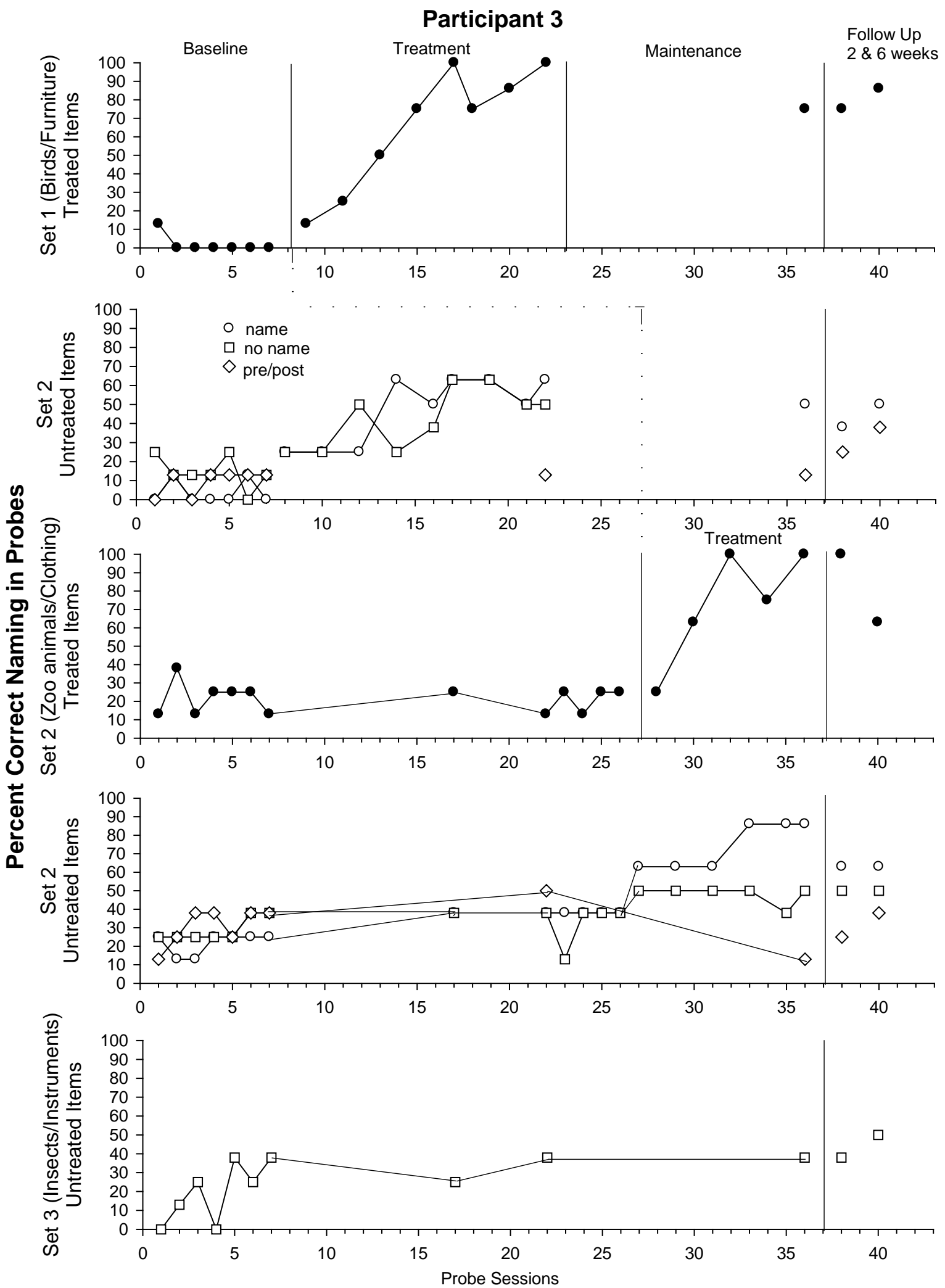


\section{Participant 4}
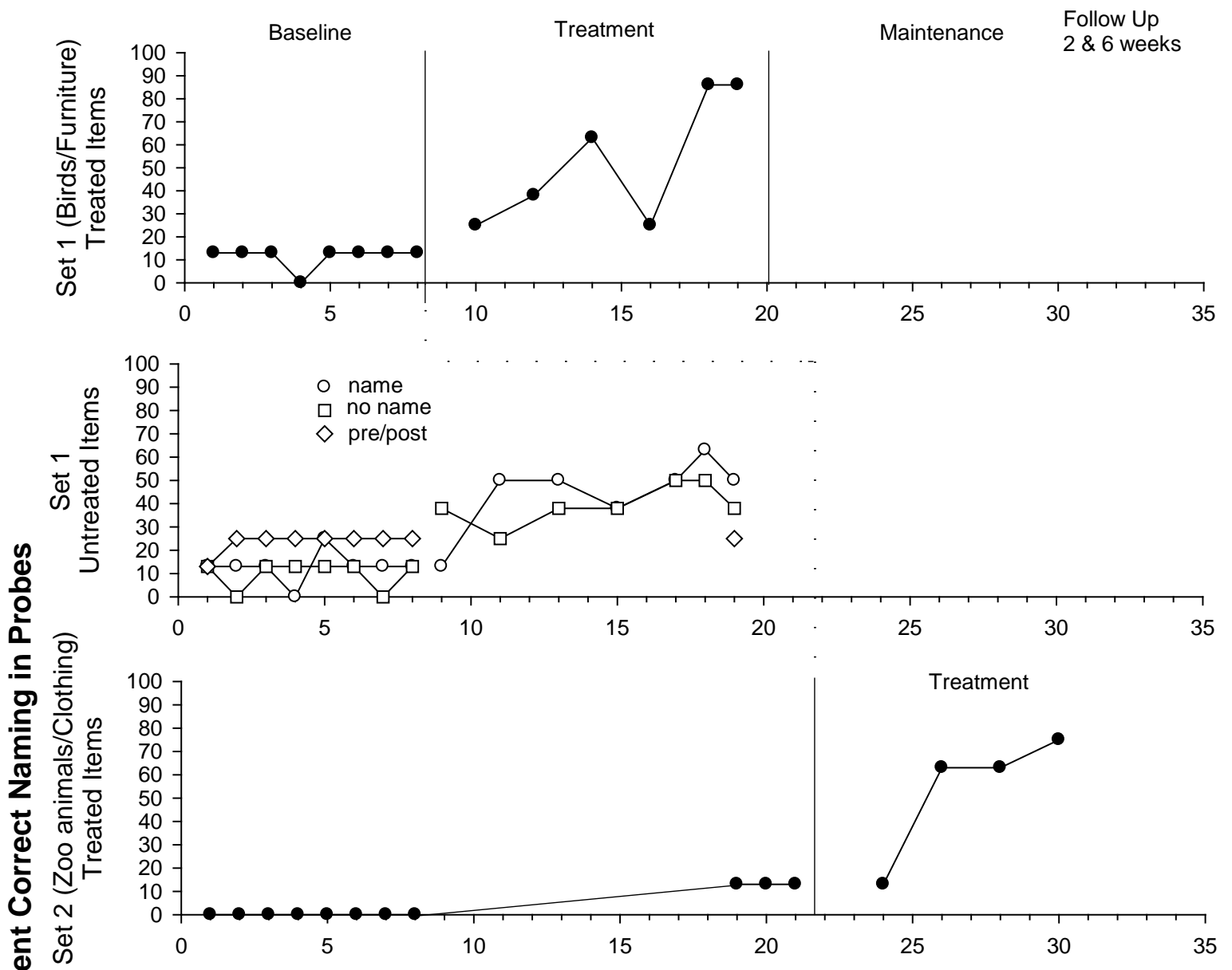

ญั
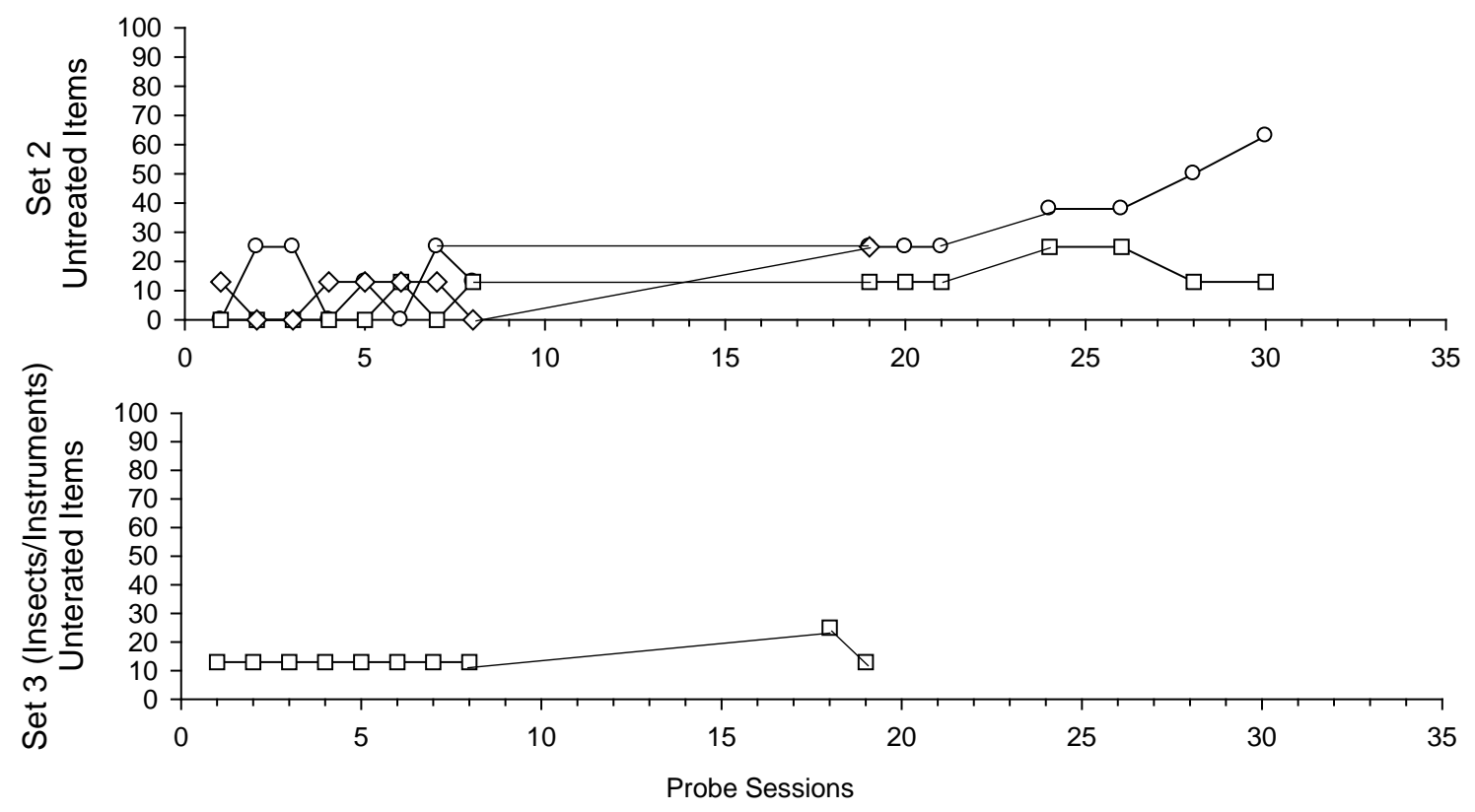
Figure 5

Semantic Information Units

\begin{tabular}{lcccc}
\hline Participant & $\begin{array}{c}\text { Pre Treatment } \\
\text { SIU/Words }\end{array}$ & Percentage & $\begin{array}{c}\text { Post Treatment } \\
\text { SIU/Words }\end{array}$ & Percentage \\
\hline Participant 1 & $168 / 780$ & $22 \%$ & $159 / 477$ & $33 \%$ \\
Participant 2 & $295 / 4,928$ & $6 \%$ & $345 / 4,553$ & $8 \%$ \\
Participant 3 & $131 / 209$ & $63 \%$ & $163 / 245$ & $67 \%$ \\
Participant 4 & $804 / 5,558$ & $14 \%$ & na & na \\
\hline
\end{tabular}


Appendix A

\section{Examples of Naming Errors for Each Participant: Responses from Baseline Probes}

\section{Participant 1}

$\begin{array}{ll}\text { Target } & \text { Response } \\ \text { magpie } & \text { bagpie } \\ \text { puffin } & \text { I don't know } \\ \text { pigeon } & \text { penguin } \\ \text { flamingo } & \text { fabingo } \\ \text { coatrack } & \text { coarag } \\ \text { stool } & \text { skool } \\ \text { crib } & \text { cwib } \\ \text { recliner } & \text { recider } \\ \text { alligator } & \text { crosadile } \\ \text { antelope } & \text { deer } \\ \text { zebra } & \text { sebu } \\ \text { hippo } & \text { hislapot } \\ \text { sombrero } & \text { soburu } \\ \text { nightgown } & \text { gown something } \\ \text { earmuffs } & \text { muff something }\end{array}$

Participant 2

$\begin{array}{ll}\text { Target } & \text { Response } \\ \text { goose } & \text { go over all the time } \\ \text { cardinal } & \text { it's the redder for the } \\ \text { parrot } & \text { pakaw } \\ \text { hummingbird } & \text { hippie or a } \\ \text { lamp } & \text { lamper } \\ \text { dresser } & \text { I don't know } \\ \text { bookcase } & \text { berbrek } \\ \text { wastebasket } & \text { bavewood } \\ \text { bat } & \text { bat } \\ \text { beaver } & \text { bavie } \\ \text { panther } & \text { liger } \\ \text { hyena } & \text { hasvena } \\ \text { belt } & \text { bess } \\ \text { mittens } & \text { muffs } \\ \text { nightgown } & \text { night time }\end{array}$

\section{Participant 3}

$\begin{array}{ll}\text { Target } & \text { Response } \\ \text { puffin } & \text { bird } \\ \text { pigeon } & \text { bird } \\ \text { flamingo } & \text { f something } \\ \text { magpie } & \text { bird } \\ \text { coatrack } & \text { I don't know } \\ \text { stool } & \text { stool } \\ \text { crib } & \text { baby bedroom } \\ \text { recliner } & \text { chair } \\ \text { zebra } & \text { zeba } \\ \text { antelope } & \text { deer } \\ \text { panther } & \text { bobcat } \\ \text { skunk } & \text { stinky } \\ \text { bathrobe } & \text { towel } \\ \text { blouse } & \text { sweater }\end{array}$

\section{Participant 4}

$\begin{array}{ll}\text { Target } & \text { Response } \\ \text { puffin } & \text { bird } \\ \text { pigeon } & \text { green bird } \\ \text { magpie } & \text { mapie } \\ \text { flamingo } & \text { flounders } \\ \text { coatrack } & \text { hangers } \\ \text { stool } & \text { stool } \\ \text { credenza } & \text { furniture put radio in there } \\ \text { recliner } & \text { swing chair } \\ \text { anteater } & \text { I don't know } \\ \text { panther } & \text { pander } \\ \text { skunk } & \text { skun } \\ \text { bathrobe } & \text { robe } \\ \text { nightgown } & \text { sleeping dress } \\ \text { leotard } & \text { leo }\end{array}$


Appendix B

\section{Participant Stimuli}

\section{Participant 1 Stimuli}

Set 1: Birds \& Furniture

1. SFA treatment items

magpie

puffin

pigeon

flamingo

crib

stool

coatrack

recliner

3. Generalization-repeated exposure items

canary

hawk

seagull

bluebird

cot

throne

hammock

chandelier

\author{
2. Naming control items \\ goose \\ roadrunner \\ cardinal \\ hummingbird \\ playpen \\ frame \\ dresser \\ wastebasket
}

4. Generalization-limited exposure items ostrich

swan

vulture

woodpecker

hutch

futon

nightstand

ottoman

Set 2: Zoo Animals \& Clothing

1. SFA treatment items

zebra

hippo

antelope

alligator

blouse

nightgown

earmuffs

sombrero

3. Generalization-repeated exposure items

skunk

rhino

giraffe

kangaroo

robe

blazer

overalls

cardigan
2. Naming control items

panther

beaver

hyena

koala

bonnet

tracksuit

miniskirt

pajamas

4. Generalization-limited exposure items

wolf

panda

raccoon

porcupine

scarf

bowtie

corset

tuxedo 
Set 3: Insects and Musical Instruments

1. Generalization-repeated exposure items

moth

cockroach

dragonfly

wasp

drum

flute

banjo

accordion

Participant 2 Stimuli

Set 1: Zoo Animals \& Clothing

1. SFA treatment items

bat

beaver

panther

hyena

belt

socks

mittens

nightgown

3. Generalization-repeated exposure items

hippo

zebra

elephant

porcupine

jacket

blouse

bathrobe

earmuffs

2. Naming control items

seal

goat

kangaroo

giraffe

vest

boots

shorts

suspenders

4. Generalization-limited exposure items

wolf

tiger

cougar

lion

purse

scarf

tuxedo

sandals

Set 2: Birds \& Furniture

1. SFA treatment items

goose

cardinal

parrot

hummingbird

lamp

dresser

bookcase

wastebasket

2. Naming control items

eagle

swan

vulture

woodpecker

stool

chair

curtain

recliner 
3. Generalization-repeated exposure items hawk

owl

crow

bluebird

pillow

crib

hammock

chandelier

Set 3: Insects and Musical Instruments

1. Generalization-repeated exposure items

spider

butterfly

ant

cockroach

guitar

drum

banjo

flute
4. Generalization-limited exposure items turkey

dove

flamingo

magpie

frame

rug

nightstand

ottoman

\section{Participant 3 Stimuli}

Set 1: Birds \& Furniture

2. SFA treatment items

magpie

puffin

pigeon

flamingo

crib

stool

coatrack

recliner

2. Naming control items

goose

roadrunner

cardinal

hummingbird

playpen

frame

dresser

wastebasket

3. Generalization-repeated exposure items

canary

hawk

seagull

bluebird

cot

throne

hammock

chandelier

4. Generalization-limited exposure items ostrich

swan

vulture

woodpecker

hutch

futon

nightstand

ottoman

Set 2: Zoo Animals \& Clothing

2. SFA treatment items

2. Naming control items 


zebra
hippo
antelope
alligator
blouse
nightgown
earmuffs
sombrero
alization-repeated exposure items
skunk
rhino
giraffe
kangaroo
robe
blazer
overalls
cardigan

3. Generalization-repeated exposure items skunk

rhino

giraffe

robe

blazer

cardigan

Set 3: Insects and Musical Instruments

1. Generalization-repeated exposure items
moth
cockroach
dragonfly
wasp
drum
flute
banjo
accordion

panther

beaver

hyena

koala

bonnet

tracksuit

miniskirt

pajamas

4. Generalization-limited exposure items wolf

panda

raccoon

porcupine

scarf

bowtie

corset

tuxedo

\section{Participant 4 Stimuli}

Set 1: Birds \& Furniture

3. SFA treatment items

puffin
pigeon
flamingo
magpie
coatrack
stool
credenza
recliner

3. Generalization-repeated exposure items canary

2. Naming control items

goose
cardinal
roadrunner
ostrich
playpen
shutters
dresser
wastebasket

4. Generalization-limited exposure items hummingbird 
hawk

seagull

bluebird

cot

throne

futon

chandelier swan

vulture

woodpecker

hutch

hammock

nightstand

ottoman

Set 2: Zoo Animals \& Clothing

3. SFA treatment items
anteater
antelope
panther
sloth
bathrobe
sombrero
leotard
earnuff

3. Generalization-repeated exposure items

otter

lemur

alligator

warthog

bonnet

blazer

overalls

cardigan
2. Naming control items

skunk

rhino

armadillo

gekko

nightgown

miniskirt

tracksuit

fedora

4. Generalization-limited exposure items panda

koala

hyena

porcupine

bowtie

corset

poncho

tuxedo

Set 3: Insects and Musical Instruments

1. Generalization-repeated exposure items

dragonfly

praying mantis

cockroach

moth

accordion

saxophone

banjo

tambourine 


\begin{tabular}{|c|c|}
\hline $\begin{array}{l}\text { Respor } \\
\text { Respor }\end{array}$ & 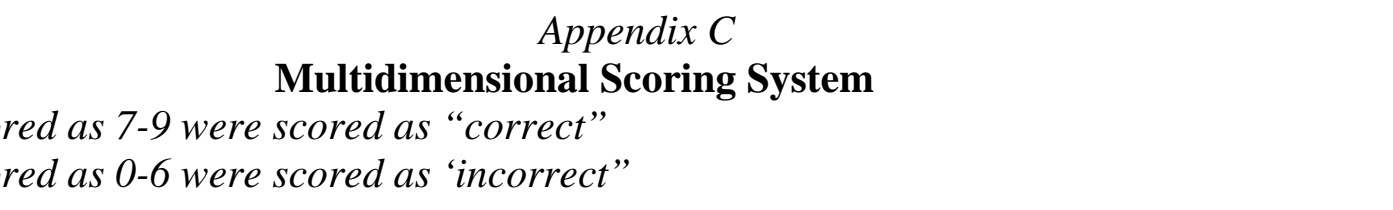 \\
\hline Score & Description \\
\hline 9 & Accurate, immediate ( $<5$ seconds) \\
\hline 8 & Accurate delayed (correct response within 6 to 20 seconds) \\
\hline 7 & Incorrect plural or singular form \\
\hline 6 & $\begin{array}{l}\text { Phonemic paraphasia (i.e., single phoneme substitution or recognizable word with } \\
\text { more than one sound substitution; at least } 50 \% \text { of sounds correct) }\end{array}$ \\
\hline 5 & Semantic paraphasia (semantically related word) \\
\hline 4.5 & Semantic paraphasia containing phonemic paraphasia \\
\hline 3 & Circumlocution \\
\hline 2 & Neologism or unintelligible word \\
\hline 1 & Perseveration (response that was used for a previously presented item) \\
\hline 0 & No response or “I don’t know” \\
\hline
\end{tabular}

Appendix D

Participant 1

\section{Example transcriptions with SIUs underlined}

1. Zebra-um he has four $\mathrm{xx}$ legs um he has um force he has um ze no white and black um xxx xx has kail a tail and something

2. Rhino- um he has four um legs he has um gray and its whsl wasl stum rough on du on du sides and um he's dut corn two forms on um two ears two hears horns das it horns

3. Tracksuit- warm warm things um a pants about uh dat has lastic on dis front and um uh button nu um zipper on du thing on whatever it's black on du arms and red on du the tings I don't know it and uh uh zipper on the front um

Participant 2

1. Wastebasket- and this is a they're called they're in your bedroom or in your be or in your house put them on in your kitchen or wh or the're bathroom floor or bathroom the baste for a basket a base a basebasket uh I can't say the namebas bask xx bascar they call them you bath your kitchen they're bathroom they call em they call em other sorts of things I can't say

2. Hippo - this one too is a himpo hippo it's a hippo it's himpio I can't say himipa I can't say it's full name casue it's too big a word hip hippo it's hippo and they live in Africa and they have lots of $x$ they h they usually have lots of they live they they live inside they don't live in on them in the water but they hang in it all the time and then they'll sleep outside they'll go out the around there with the um hi like the hipir oh now I can't think or their name again hi hippo hip he's a hippp hipia yeah ok and um they just eat they usually just eat the food that they er this jus they usually just eat um what uh food er uh what kinds of things can't think iof the name how to feed them anyway ok 
3. Belt- these are they're called they're called for th they are called for a bek you wear them on your clothes you put them on your pants to hold them up and you call em they are called they er ba I can't say their name they're um you hold em with their with your be your bre your bleaks I can't think of the name though

Participant 3

1. Cot- army tent sleeping

2. Woodpecker- bird uh worms scared of man and woman

3. Tracksuit- mens sweatpants and sweatshirt training weights fitness

\section{Participant 4}

1. Bathrobe- ok this um is a bathrub uh bathrobe and this is um you put like you put in your either before or after just sleep or just sleep before and after sleep and makes ya um like you have a shower nice and warmer or or when you get up put ya nice and warm when you get your breakfast or whatever $\mathrm{x} x$ warm at the time

2. Panda- ok this is a um panda and um it's white and black well it the face looks like looks like a bear it big and fat and um small ears and um the face the face is all white but the ears and eyes are black and the little nose is black and then it then it's face like white and black with the middle part er the legs are all black and the back part is white and um it's um it's from at the zoo or it's from I can't think what it's from um it's a different country now I can't remember exactly where the country though

3. Dragonfly- this is like a um like a butterfly but it's not a butterfly but it's but um but it flies uh he's got four um it's like a butterfly I don 't know what it's called though but it flies on that or it can walks and it's kinda on the on the um um I don't know what these are called though but it's not the arms though anyway but it's clear but the face is green and the body is really long and thin and it's kinda blue 